\title{
ESTIMATING THE SUPPLY CHAIN EFFICIENCY LOSS WHEN THE SELLER HAS TO ESTIMATE THE BUYER'S WILLINGNESS TO PAY
}

\author{
XAVIER BRUSSET ${ }^{1}$
}

\begin{abstract}
We study the pricing problem between two firms when the manufacturer's willingness to pay (wtp) for the supplier's good is not known by the latter. We demonstrate that it is in the interest of the manufacturer to hide this information from the supplier. The precision of the information available to the supplier modifies the rent distribution. The risk of opportunistic behaviour entails a loss of efficiency in the supply chain. The model is extended to the case of a supplier submitting offers to several manufacturers. Some managerial insight through a numerical illustration is provided.
\end{abstract}

Keywords. Supply chain management, information asymmetry, Bayesian belief, mechanism design, log-concave distributions.

Mathematics Subject Classification. 90B50, 91A40, 91A10, 91A28.

\section{INTRODUCTION}

In a supply chain and particularly in B2B markets, firms often have to look for new customers and new markets for their products. Motivation for the present paper can be found in the way that some suppliers have to price some specialised good or service which they sell to some manufacturer without being fully informed of the value of this good or service to this manufacturer, nor the potential competitors' price for similar product. Usually, the supplier already has a steady client base through which to sell the same good. They can be in different markets for different

Received June 3, 2013. Accepted March 17, 2014.

1 ESSCA School of Management, Center of expertise and Research in Retailing, 55, Quai Alphonse Le Gallo, 92513 Boulogne Billancourt Cedex, France. xavier.brusset@essca.fr 
uses and at different prices. For example, agricultural, chemical or mineral products have wide ranging applications: calcium carbonate is used in industries like paint, plastic, rubber, ceramic, cement, glass, steel, oil refining, iron ore purification and biorock creation for mariculture of sea organisms ${ }^{2}$. Gelatin from organic material has applications in pharmaceutical products, X-ray and photo prints, cosmetics, banknotes and food among others. Road transport in its common form can transport any type of common cargo. Enterprise Information Systems can be tailored to suit different customers in different industries. More generally, some products have very different uses but essentially the same production process and cost.

In most of the above instances, the exact relationship between demand in these new markets and price may not be known. In fact, even after accounting for the intrinsic qualities of his product, the supplier still has to guess at his potential clients' willingness to pay (wtp) or reservation price. To do so, he must build upon his prior knowledge of the industry to which the prospective client belongs, the potential existing competition, alternative sources of supply, etc. It may also induce a less efficient supply chain in a dynamic setting as shall be shown later. This lack of information may induce unsatisfactory pricing decisions leading to either unsuccessful offers or less profitable transaction for the supplier. It may further stunt the supplier's effort in developing the product into a more specific one better suited to the new customer.

On the other hand, the manufacturer will usually hide or mis-inform the supplier about his wtp. The prospective clients use such input in their industrial processes to enhance either the quality of the final end-product or the efficiency of the production process.

Consider the use of calcium carbonate as paper coating or as water absorbent for diapers: the multiplying factor in value for the supplier between the first and second use is a power of ten ${ }^{3}$. Some twenty years ago, it was found that when fine grains of calcium carbonate were flash calcined (cooked for a fraction of a second at a very high temperature), the grains exploded into balls that could absorb several times their volume in water. Kimberley Clarke was approached with an offer to produce such grains in sufficient volume if they could be incorporated into diapers. The kiln to flash calcine the required volumes is an expensive piece of equipment. Either Imerys or Kimberley Clarke could have invested in such equipment. However, since the expertise in rock processing lies more with Imerys, the dyad makes a higher rent if Imerys invests than if the downstream partner does. The decision and the subsequent rent hinged on the paper maker buying the final product at a much higher price than for traditional markets like paper coating.

\footnotetext{
${ }^{2}$ See the entry in Wikipedia on the industrial applications of calcium carbonate. The AngloFrench corporation Imerys has two separate business units which refine and sell this mineral for separate uses but essentially the same production processes.

${ }^{3}$ The reader will find further technical information at: http://www.imerys-perfmins.com/calcium-carbonate/eu/calcium-carbonate-film.htm
} 
In most research on supply-chain contracting a common assumption is that all relevant information is common between the parties concerned [8].

How is the supplier to price his good? What is the effect on supply chain rent?

We answer these questions using a mechanism design approach in a games theoretic setting where a principal wishes to set a price for some product or service he wishes to sell to an agent. If the agent rejects the offer, the principal is left with the revenue generated from selling to a non-strategic third party. The supplier is in a Bayesian setting of incomplete information ${ }^{4}$ and must form a belief about the agent's wtp. The setting of this problem is well known and has been amply covered in literature. The model shows how the supplier maximizes his profit by his pricing decision and how the manufacturer will attempt to increase his rent in detriment of the supplier by keeping information private. The model yields insights into two consequences: (a) information sharing by the downstream partner leads to opportunistic behaviour on the upstream party's part; (b) effort to increase the rent of the supply chain will probably be misplaced or not undertaken leading to long-term inefficiencies in the supply chain.

The present model helps to present in a new light the newsvendor one from [25] in which the single price contract is studied when both manufacturer and supplier know of the distribution of demand and the sensibility to price of this demand.

The paper is organised as follows. In the next section, we present a brief review of existing literature justifying our approach. The model is explained in Section 3. Two theorems are presented: in Section 3.1 for the univariate case and in Section 3.2 for the multivariate one where a supplier faces several manufacturers with unknown wtp. In Section 4, we illustrate the use of the first theorem when comparing an integrated to a decentralised supply chain management. In Section 4.1, we prove that the agent will obfuscate information about his wtp in an attempt to prevent the principal from behaving opportunistically when pricing his product. In Section 4.2, we prove how the information asymmetry can induce misallocation of effort and investment and hamper supply chain rent creation. The corresponding insights are further illustrated succinctly in Section 5 with an upstream agent who has to deal with first one and than two downstream customers. We conclude in Section 6.

\section{LiteratuRE REVIEW}

Game Theory has been widely used in supply chain management [27]. The different parties in a supply chain are called players in game theory. The profit function of each player is called the payoff function. Our model fits into the stream of supply chain literature which builds upon non-cooperative Game theory using pure strategies as reviewed in $[10,26]$. According to the classification introduced in $[18,19]$,

\footnotetext{
${ }^{4}$ We only consider here the complete information Bayesian games ( $C$-games) in which both players know each other's payoff functions (i.e., rules of the game and strategy spaces) and his attributes as defined in $[21,22]$ and will henceforth only take into account the imperfection of information which one player may suffer about the other player and name it incomplete.
} 
our model fits in the category of static games with incomplete information leading to a Bayesian Nash Equilibrium. Some insights into dynamic interaction between buyer and seller are also given.

The mechanism design presented here builds along the seminal work depicted in [17] where the seller elicits the types of the buyers (through the revelation principle) and hence chooses his rent maximising transfer mechanism. The revelation principle has been used in several settings both in game theory and supply chain management theory. The revelation of information is basically achieved when the seller offers a menu of contracts from which the buyer must choose. In so doing, the relevant information important to the seller is revealed. Let us cite some of them here. The buyer's set-up cost of ordering can be revealed as shown in [15]. Reference [13] shows how asymmetric information about market demand can be obtained by the seller. [11] shows how a seller can elicit the market condition known to the buyer but not the seller. [20] deals with asymmetric cost information. [14] shows how the seller can obtain the buyer's back-order cost. Note that only one type of information can be revealed this way unless the seller were to offer not just a single list of contracts but a two- or three-dimensional matrix of contracts. Even if elegant mathematically, this becomes inconvenient in practice. Moreover, the case where the seller is limited by the buyer into submitting just one contract is not addressed. In the case of Imerys, it would have been confusing and impractical to submit a menu of contracts to Kimberley Clarke to implement the economic interaction of selling calcined kaolin for diaper manufacturing. In the same vein, if a refrigerated lorry fleet operator usually carrying food receives a request to quote from a pharmaceutical manufacturer, an industry unknown to him, submitting a menu of contracts is not relevant.

Other models often presented in literature involve bargaining. One player makes offers and expects the other player to either accept or turn it down in which case the first player makes a better offer, renewing this back and forth until an agreement is finally reached. As shown in [30], the player making the offer can extract useful information from the answers of the other player and build upon it to maximise his payoff function. Here again, the situation we are trying to describe does not fit the bargaining model especially as neither players possesses information about the other's cost and opportunity types. The Imerys commercial representative is not aware of the extra value that his product will bring to the Kimberley Clarke diapers, Kimberley Clarke is unaware of Imerys production cost or its other customers' buying price.

Still other models consider signaling games in which the buyer signals his wtp to the seller [9]. This supposes that the buyer has an interest in helping the seller because not doing so reduces his payoff. In our motivating examples, the buyer can rely on third parties to satisfy his need, reducing the advantage of signaling. We will see however that the buyer still has an interest in conveying some type of information, but the purpose is to deceive the seller, not increase both player's overall payoff. 
The problem presented in introduction was numerically solved in [6,7] as particular instances of the much broader mechanism design problem presented here. A similar model is presented in [33] further simplified in [24], the example cited is of a service's pricing. The model in [24] considers that the seller knows the demand function and optimizes his price accordingly. In the present paper, we consider that the seller is uninformed of the demand he will face and hence is unaware of the manufacturer's wtp.

Reference [3] (first put out as a working paper in 1989) presents a number of theorems and properties based upon Log-concave and Log-convex distributions. [17] use it to show a famous result in auction theory [32]. Namely that, under some assumptions, the first- and second-price auctions yield the seller the optimal expected revenue when the buyers are symmetric, have independent valuations and have a continuum of potential valuations. The characterization of the single optimal allocation for a principal of a good to one buyer among several also uses the fact that Log-concave distributions have increasing failure rates.

The models presented in [25] or in [34] also involve a manufacturer selling to a newsvendor through a single price contract and studies the impact of demand variability and level of the price on the buyer's effort and profit. The model in [33] studies the optimal admission price to a service facility for customers who have a known wtp distribution function. The model in [29] refines the newsvendor models of $[24,25]$ by offering some restrictive conditions so that the manufacturer is guaranteed to have a unimodal profit function.

Yet in all of the above models alternative sources or lack of information about the buyer's wtp are not included in the objective functions. To our knowledge, the case where the seller is supposed to make one single offer to a buyer without being aware of this buyer's wtp nor of alternative sources and must rely on some a priori belief about this buyer's wtp has not been addressed. The present model and its discussion is a contribution towards closing this gap in literature.

\section{MODEL}

A supplier has the ability to make a product or deliver a service that can have applications in widely differing areas and hence be of interest to very different customers. This heterogeneity in cost structures, payoff functions, and demand for final products on the part of customers makes it difficult to the supplier to be precise in his pricing offers. Let us consider that a supplier already has an existing non-strategic customer to which he sells his product or service. On the other hand, a manufacturer requires this product or service for his own production but has an existing non-strategic alternative supplier for a similar or substitutable product or service. The supplier and manufacturer have not yet traded this particular good or service before. In time, we position ourselves after the discovery process by either has terminated but before any trading offer has been made. If it is the manufacturer who discovers the supplier, then he sends a request for a quote (Request For Quote (RFQ): very common practice in the case of tenders). If it is 
the supplier who discovers that the manufacturer could have a use for his product or service, he makes an unsolicited offer. In what follows, we shall consider this initial offer by the supplier to be the first step of the game and hence consider the supplier as Stackelberg leader. The supplier has the capacity to make one product or service which is why he has to choose to who he sells this product or service to. Given his profit-maximising objective, he will sell to the highest paying customer. No inventory will be left over as either the potential new customer or the existing one will receive the product.

The model presented here considers that the supplier can sell to the manufacturer or to an alternative customer at a different price. It can accommodate both a supplier addressing an offer to a single manufacturer as well as the case where he can make simultaneous offers to several customers for which he may hold distinct Bayesian beliefs about their wtp. These beliefs are assigned the same distribution function but with distinct parameters. We start with the single potential client case before extending the results to the multi-client one.

As is often the case in practice, we consider that the seller will use a contract specifying only one price or a simple list linking price to quantity (in difference to the menu of contracts mentioned above, this is a take it or leave it offer: the buyer cannot pick). This contract is not within the scope of the coordinating or collaboration mechanisms usually described in literature $[2,16,23,31]$. One justification can be that other managerial coordination processes will be set up once an agreement has been reached.

\subsection{Case of a single potential Customer}

The supplier, as principal and Stackelberg leader, is uninformed of the buyer's reservation price for a good he wishes to sell. If he guesses wrongly this price, he can still dump his good on a non-strategic third party for a price $\alpha$. The supplier has to form a Bayesian belief about the distribution of this reservation price and assign it a subjective probability distribution.

Let $X$ represent the principal's belief about the agent's reservation price as a random variable with cumulative distribution function (CDF) $F$ ranging over $[\underline{X}, \bar{X}]$, continuous and twice differentiable. By convention, let $\bar{F}(x)=1-F(x)$. Let $f$ be its probability density (PDF). We assume without loss of generality that $0<\underline{X}<\bar{X}$. This distribution's failure rate function as defined in [4] is $r(X)=f(X) / \bar{F}(X)^{5}$. X has an increasing failure rate (IFR) or, equivalently, $F$ is an IFR distribution if $r(X)$ is weakly increasing for all $X$ such that $F(X)<1$. As shown in Appendix Section A.1, given the domain we are interested in, these functions belong to a wide family of distribution functions named Log-concave. A property of these functions is that the set $\{x \mid f(x)>0\}$ is connected. We define $\alpha$, a positive real, as the price received by the principal when the buyer refuses the offer and

$$
0<\underline{X} \leq \alpha \leq \bar{X}
$$

\footnotetext{
${ }^{5}$ This function is also called hazard function.
} 
Note that if that were not the case, the supplier's belief would have no bearing on his objective function. If $\alpha<\underline{X}$, the maximum revenue for the principal is achieved for him by choosing $\underline{X}$ as the offering price. Similarly, if $\alpha>\bar{X}$, the principal just sells to this third party for $\alpha$ and does not consider selling to the agent.

Tracing a parallel to the model in [24], here the supplier also has to sell to a downstream partner (say a retailer) a quantity at a posted price. However, in our setting, the supplier can also sell to a third party in the case where the quoted price does not satisfy the manufacturer. This option can also be seen as the buyer's option of returning all unsold goods to the supplier. Contrary to the present setting, in [24] the supplier bears no responsibility for the unsold goods and enjoys full information about the retailer's demand and retail price.

Here, the supplier does not have information about the manufacturer's reservation price nor about the competition's eventual offer, so the supplier, based upon his belief, must maximise the following objective function

$$
\begin{aligned}
\Pi_{s}(x) & =\alpha F(x)+x \bar{F}(x), \\
& =F(x)(\alpha-x)+x,
\end{aligned}
$$

after normalising the cost to 0 . The case where $\alpha=0$ is the one covered in [24]. It is straightforward to prove that there is a unique interior point within the range $[\underline{X}, \bar{X}]$ (the proof can be found in Appendix A).

The manufacturer's objective function is more straightforward:

$$
\Pi_{m}(x)= \begin{cases}p-x, & \text { if } \mathrm{x}<\gamma, \\ p-\gamma & \text { if } \mathrm{x} \geq \gamma,\end{cases}
$$

where $p$ is the expected revenue to be generated through the use of the good or service provided either by the supplier or the non-strategic alternative source and $\gamma$ is the alternative source's cost for this good or a substitute.

Some corollaries can be stated stemming from the properties of the first differential of the seller's profit function.

Corollary 3.1. If $F$ is such that $F(1)=1$, then $x=1$ is solution and is also a maximum because $\Pi_{s}^{\prime \prime}(1)<0$. This covers the case when the properties of the IFR distributions cannot be applied since at $x=1, r(x)$ is not defined. Similarly, if $f(\underline{X})=0$, then $\underline{X}$ is a maximum if $\underline{X} \leq \alpha$ because $\Pi_{s}^{\prime \prime}(\underline{X}) \geq 0$.

Corollary 3.2. Since $F$ is increasing, the domain $[\underline{X}, \bar{X}]$ can be truncated at the largest value $x_{1}$ for which $f\left(x_{1}\right)=0$ and set $\underline{X}=x_{1}$. So when $x=\underline{X}, \Pi_{s}(x)=\underline{X}$.

We enunciate the following theorem.

Theorem 3.3. Assuming that $F$ is log-concave with a finite support $[\underline{X}, \bar{X}]$, then the seller has a unique optimal solution $x^{*}$ to his concave profit function which is solution to

$$
x^{*}-\alpha=\frac{\bar{F}\left(x^{*}\right)}{f\left(x^{*}\right)} \text {. }
$$


Corollary 3.4. The optimal value is always higher than the outside option price $\alpha$ reflecting the belief held by the seller that there is a non-zero probability that the buyer is willing to pay more than $\alpha$.

\subsection{Case of Several potential Customers}

Up till now, we have been looking at the univariate variable case. Let us now solve for the case where the supplier, having one single good to sell, may wish to offer it to several customers, all of which would have different wtp. We build on properties of log-concave distributions as characterised in $[1,4]$ and as further elaborated in [3]. We extend some of their properties as described in Appendix in A.1. These properties can be applied in cases where the upstream party has to price a good or service to $k$ potential customers involved in different industries and hence where the beliefs about their outside options may be different. In this section, we consider that the supplier has to build a belief about $k$ wtp and assigns such overall belief to a multivariate random variable $X_{K}$. This multivariate random variable has support in a $\mathrm{CDF} F_{k}($.$) and multivariate density function f_{k}($.$) .$

Let $X_{K}=\left\{X_{1}, X_{2}, \ldots, X_{k}\right\}$ be a $k$-dimensional continuous random vector with density function $f_{K}(X)$ and $\operatorname{CDF} F_{K}(X)$.

Define $S_{K}(x)=P\left(X_{K}>x\right)$.

Proposition 1 of [1] states that if $f_{K}(x)$ is log-concave, then

$r_{K}(x)=f_{K}(x) \overline{F_{K}}(x)$ is mildly increasing, i.e., $r_{K}^{\prime}(x) \geq 0$.

Proposition 4 of that same source extends this result to the multivariate case by stating that if $f_{K}(x)$ is log-concave on its support, then

- Both $F_{K}(x)$ and $S_{K}(x)$ are log-concave.

- The marginal densities of all sub-vectors are log-concave.

- $f_{K}(x)$ is unimodal.

The above results enable us to extrapolate the results for the univariate world to the multivariate one. In particular, a positive valued $k$-dimensional continuous random vector with log-concave density function is IFR.

Let us now see how this can apply to our field.

Consider the case of a supplier who can sell the same good to either an existing non-strategic customer or to $k$ potential customers. The case where the potential customers can act strategically has been amply covered in [17]. Customers may be considered to be non-strategic if they do not know each other as in our motivation examples where they hail from different industries. He lacks information about their wtp and so assumes that the corresponding $X_{1}, \ldots, X_{k}$ random variables for each customer's wtp have finite positive supports $\left[\underline{X}_{i}, \bar{X}_{i}\right], i=1, \ldots, k$ and can be represented by density function $f_{i}($.$) taken from the same family of log-concave$ distributions but with distinct parameters. The $X_{i}$ variables may be correlated for which the covariance will be part of the calculation. Jointly, these variables can be described by a $k$-dimensional continuous random vector with multivariate density function $f_{K}(x)$ and a cumulated density function $F_{K}(x)$. The supplier can offer to all potential customers his only good at one single price which allows him 
to maximise his profit according to his Bayesian belief. All potential customers announce simultaneously their acceptance or refusal decisions. Only one among those who decide to buy will be satisfied. If several customers accept the offer, the supplier chooses one of these and the others will fall back on their outside options. This case is not addressed here. In the real world, the supplier might be able to subcontract another supplier to sell to these willing customers or increase his own quantity on offer ${ }^{6}$. If no potential customer decides to buy, the supplier falls back on his non-strategic outside option. His profit function can be written as

$$
\Pi_{s}(x)=\alpha F_{K}(x)+x\left(\overline{F_{K}}(x)\right)
$$

where $x$ and $\alpha$ are scalars. He sells only one product to just one customer either at $x$ if at least one buyer accepts his offer or at $\alpha$ to the existing non-strategic partner. This profit function can be compared to the one in the univariate example as presented in (3.2).

We now enounce the following theorem.

Theorem 3.5. Let $X$ be a $\mathrm{k}$-dimensional continuous random vector of random variables $X_{1}, \ldots, X_{k}$ with joint density function $f_{K}(x)$ and joint $C D F F_{K}(x)$ with the marginal densities' finite support being $\left[\underline{X}_{1}, \bar{X}_{1}\right], \ldots,\left[\underline{X}_{k}, \bar{X}_{k}\right]$ and $\forall i, 1 \leq i \leq$ $k, \underline{X}_{i} \geq 0$. It exists a unique optimal solution $x^{*}$ to his profit function which is the solution to

$$
x^{*}-\alpha=\frac{\overline{F_{K}}\left(x^{*}\right)}{f_{K}\left(x^{*}\right)},
$$

if $F_{K}$ is a multivariate log-concave distribution.

Proof. The proof follows along the same lines as in the single variate case.

We have answered the question in introduction about how a supplier should price his good in the face of the demand from one or several downstream parties without knowing their wtp. We now turn to the second question: what is the effect on supply chain efficiency?

\section{Supply CHAIN EFFICIENCY}

To answer such a question, we first analyse the impact of the precision of information and then the lack of, or inefficient, investment in enhancing the overall rent of both partners.

The study of the efficiency of the supply chain in the present scenario of unknown wtp on the downstream party's part is the study of the misallocation and the ensuing misuse of the rent generated in the supply chain by the interaction between both parties. Sub-efficiency arises from asymmetric information and opportunistic behaviour.

\footnotetext{
${ }^{6}$ This might be the case of a road transport provider who can sub-contract or lease more units to satisfy the demand.
} 


\subsection{INFORMATION OBFUSCATION BY THE MANUFACTURER}

To establish a performance benchmark, we analyse the case of an integrated firm and common information. Let $\Pi_{i}$ represent the integrated channel profit, $\Pi_{i}=\Pi_{s}+\Pi_{m}$ with the subscript letters $s$ and $m$ representing the profits to the supplier and manufacturer. We define for this scenario the manufacturer's reservation value of the good or service bought from the supplier as $p, p>0$ and an alternative sourcing price for the same good or service from a non-strategic third party $\gamma$, with $\gamma \leq p$. Each party has the opportunity to source or sell outside the organisation if that opportunity yields a larger overall profit to the supply chain. In this case, the supplier knows the manufacturer's wtp and adjusts $x^{*}=\gamma$ so that the parties have the following profits according to the respective cost of $\gamma$ and $\alpha$ to the manufacturer. We consider that the supplier's cost is normalised to zero.

$$
\begin{gathered}
\alpha>\gamma \Rightarrow\left\{\begin{array}{l}
\Pi_{m}(x)=p-\gamma \\
\Pi_{s}(x)=\alpha \\
\Pi_{i}=p-\gamma+\alpha
\end{array}\right. \\
\alpha \leq \gamma \Rightarrow\left\{\begin{array}{l}
\Pi_{m}=p-\gamma \\
\Pi_{s}=\gamma \\
\Pi_{i}=p
\end{array}\right.
\end{gathered}
$$

The case where $\alpha>\gamma$ is a trivial one: if the manufacturer is able to find an alternative source for the product or service by the supplier which is cheaper than the alternative selling opportunity that the supplier faces, both turn to their alternatives and the supply chain's integrated profit is enhanced but not based on its integration. In what follows, we focus on $\alpha \leq \gamma$, i.e., the terms of trade are favourable.

We now turn to the decentralised supply chain where the supplier is not informed of $\gamma$ or $p$.

If the manufacturer accepts the supplier's offer, it is because $x^{*} \leq \gamma$ and the overall profit to the supply chain is the same as in the integrated, common information case, i.e., $\Pi_{i}=\Pi_{d}=p$.

The manufacturer refuses the supplier's offer when $x^{*}>\gamma$ and so the supply chain's profit becomes

$$
\Pi_{d}=p-\gamma+\alpha
$$

and $\Pi_{d} \leq \Pi_{i}$ because $\alpha \leq \gamma$. Hence there is a risk of a lower profit when information is asymmetric. This risk is proportionate to the inaccuracy of the belief held by the supplier. 
To the supplier, the cost of the information is reflected in the difference in profit between the integrated $\left(\Pi_{s i}\right)$ and decentralised scenarios $\left(\Pi_{s d}\right)$ :

$$
\Pi_{s i}-\Pi_{s d}=\gamma-\alpha F\left(x^{*}\right)-x^{*} \bar{F}\left(x^{*}\right),
$$

with $\Pi_{s i}-\Pi_{s d} \geq 0$, since $\alpha<x^{*} \leq \gamma$.

It can be proven that for two log-concave random variables $F_{1}\left(\mu_{1}, \sigma_{1}\right)$ and $F_{2}\left(\mu_{2}, \sigma_{2}\right)$ built from the same distribution type, if

$$
\mu_{1}=\mu_{2}, \forall\left\{\sigma_{1}, \sigma_{2}\right\}, \text { and } 0<\sigma_{1}<\sigma_{2}<\mu / 4, \quad \Rightarrow \quad x_{1}^{*}<x_{2}^{*} .
$$

Further, the difference $x^{*}-\gamma$ tends to 0 as the first moment $\mu$ of $F($.$) tends$ to $\gamma$ and as the second moment $\sigma$ tends to 0 . In other words, if we consider that the precision of information available is continuous ${ }^{7}$, then, as the supplier becomes better informed, the difference between the integrated and decentralised chains becomes smaller. Given opportunity for mutually beneficial trade, supply chain efficiency increases with the availability and precision of information about a manufacturer's wtp.

As a corollary from equation (4.3), in the decentralised supply chain, the manufacturer's rent increases with the standard deviation of the supplier's belief distribution. In other words, the manufacturer will tend to refrain from informing or signaling to the supplier about his true alternative options expecting the optimal $x^{*}$ to be low compared to his outside option. The supply chain's overall rent may be unchanged but the profit is allocated to the downstream party.

This result is illustrated in the numerical example below (see Sect. 5.1).

\subsection{RENT MisAlLOCATION AND WASTEFUL INVESTMENT}

Having shown how information precision affects rent allocation, we now describe the mechanism by which the manufacturer, by his opportunistic behaviour in obfuscating information about $\gamma$, influences the overall rent generated from their mutual interaction.

The following reasoning assumes without loss of generality that the supply chain partners are specialised and hence that each has unique capabilities to invest effort into increasing the value of their product or decreasing the cost of their processes.

This mechanism design analysis can be applied as long as the principal and the agent can commit intertemporally to interact several times see e.g. [5]. The decisions and results from these decisions, if both wish to trade, take place as in Section 1.

The returns from investing an amount $\epsilon$ of effort by either party accrues only to that party. The decision about how much effort to provide is made considering the price $x^{*}$ and extra returns. The larger the price $x^{*}$, the more effort the supplier will put in, since the expected extra cost reductions can be potentially larger. Note

\footnotetext{
${ }^{7}$ Without loss of generality, this case can be extended to the situation where information is granular or discontinuous.
} 


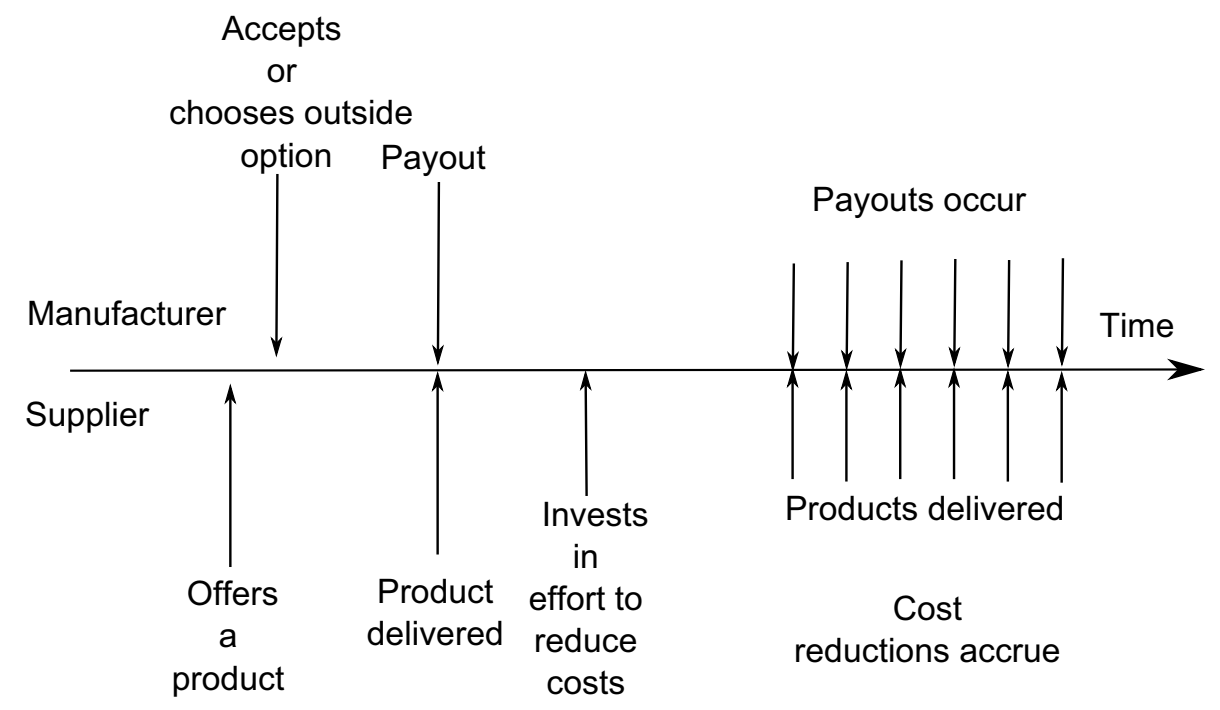

Figure 1. Timeline of decisions and events if the manufacturer decides to buy from the supplier.

that this way of ensuring effort towards the global rent generated within the supply chain is reminiscent of the way [25] models how the decrease in the wholesale price that the manufacturer charges the retailer enhances the possibility for this retailer to increase the effort in market forecast and information sharing about demand.

If, due to the Bayesian belief of the supplier, $x^{*}$ is low, the rent is displaced into the manufacturer's pocket. Because of this added rent, the manufacturer will be the one deploying an effort $\epsilon$ into increasing the chain's efficiency. But, since we hypothesise that the supply chain partners are specialised, for the same effort, the accrued cost reductions will be less than those that could have been generated by the supplier:

$$
r_{s}-\epsilon>r_{m}-\epsilon>0 \Rightarrow \Pi_{s-\text { effort }}+\Pi_{m}>\Pi_{s}+\Pi_{m-\text { effort }},
$$

with $\epsilon$ the effort provided either by the supplier or by the manufacturer, $\Pi_{s-e f f o r t}$, $\Pi_{m-e f f o r t}$ the profit accruing to the supplier or the manufacturer respectively, and $r_{s}, r_{m}$ the discounted cost reduction that accrue to each partner respectively after the effort has been provided in the transactions that follow.

We collect the result of this demonstration in a last theorem.

Theorem 4.1. In a multiple interaction setting with intertemporal commitment, the supply chain's efficiency is adversely affected by the downstream party's ability to hide or mis-represent information about his wtp to the upstream party.

As a corollary, let us mention another potential consequence. Once the supplier has found a "new" application for his product, he will wish to enlarge his customer 
base by offering it to other customers. The existing one becomes the non-strategic alternative and the supplier can update his belief of the wtp of the new potential customers using information gleaned from the first one. The supplier may hence sell his product for a higher price, depriving the manufacturer of the product.

This result is the mirror from the one recorded in [25] where the upstream partner foregoes some rent so as to enhance the downstream partner's faculty to enhance the supply chain's efficiency by decreasing the wholesale price charged to this downstream partner.

\section{Numerical illustration}

\subsection{IMPACT OF INFORMATION PRECISION}

Let us examine how the supplier's optimal $x^{*}$ based upon a belief which follows a Normal distribution would behave. Suppose that $\gamma=8$ and $\alpha=1$. In Figure 2 we can see that the optimal quantity decreases before increasing again as $\sigma$ decreases. The fact that $x^{*}$ is "high" for high values of $\sigma$ can be put down to the fact that the belief distribution spans an area much larger and includes a larger probability of values for which the alternative $\alpha=1$ is more interesting. In other words, the alternative becomes a bulwark which helps the supplier in case the manufacturer has an alternative which is higher than $\alpha$. The other conclusion is that $x^{*}$ converges to $\gamma$ as $\sigma$ tends to 0 . The standard deviation of the belief distribution is a proxy to the precision of the supplier's information.

\subsection{Illustration of the Single potential Customer CASE}

Let us illustrate the result with two different distributions. The first is a uniform continuous distribution on the range $[1,8]$. The second is an extreme value distribution with parameters with location parameter $\alpha=1$ and scale parameter $\beta=8$. In both cases, the outside option $\alpha=4$. The graphs in Figure 3 represent the corresponding profit functions and optimal values $x^{*}$.

From Theorem 3.3, we obtain $x^{*}=6$ for the uniform distribution and $x^{*}=$ 12.9671 for the extreme value distribution, both of which are higher than the outside option price $\alpha=4$ and effectively represent the maximum of the profit function.

\subsection{Illustration of the CASE With TWO POTENTial CUSTOMERS}

In the same way as above, we represent the profit function of a supplier who wants to sell the same good to two different manufacturers. He forms two Bayesian beliefs about their wtp and builds a joint distribution of their behaviours. The joint distribution is based upon the binormal distribution of the two random variables. Let us consider that $\mu_{1}=5, \sigma_{1}=0.5, \mu_{2}=8, \sigma_{2}=1$. The correlation coefficient, set at $\rho=0.9$, signals that he believes his customers to be in the same market or responding to the same signals in the same way. In this example, varying the 


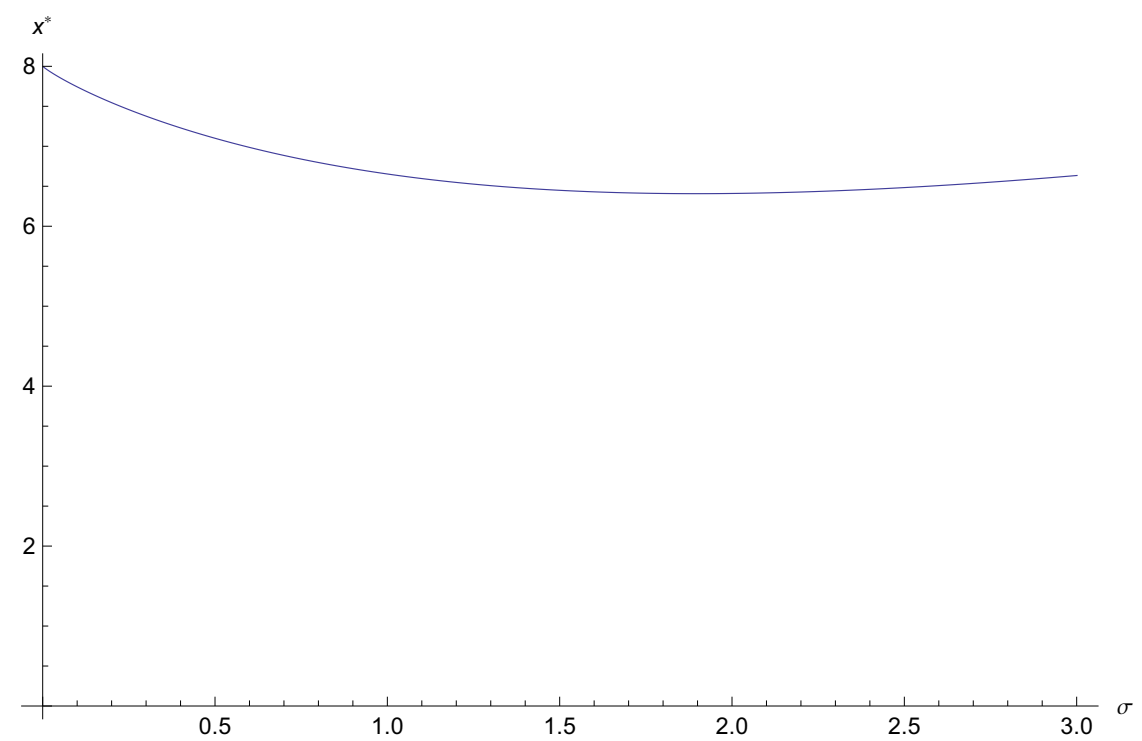

FiguRE 2. Representation of the optimal solution for the supplier as his information about the true value of the alternative option to the manufacturer becomes more precise. $\gamma=8$ and the alternative $\alpha=1$ in this example where the belief follows a Normal $(8, \sigma)$.
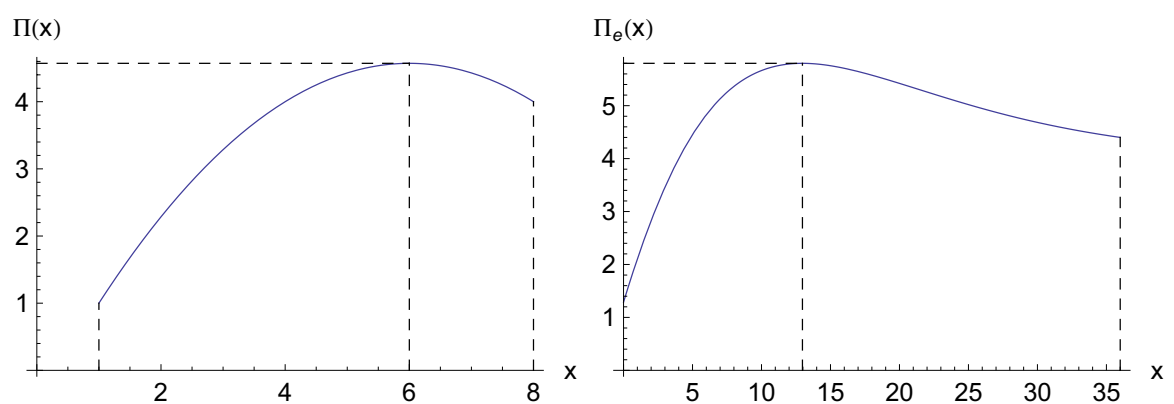

FIGURE 3. Representation of the profit function and optimal solution when the belief distribution is uniform and ranges between 1 and 8 (left graph), when the belief distribution follows an extreme value distribution (right graph) and $\alpha=4$.

correlation coefficient does not change significantly the optimal price offered by the supplier.

The outside option of the supplier is set at $\alpha=4$.

In the second case,the distributions are extreme value distributions characterised by parameters $\{1,8\}$ and $\{2,10\}$. 

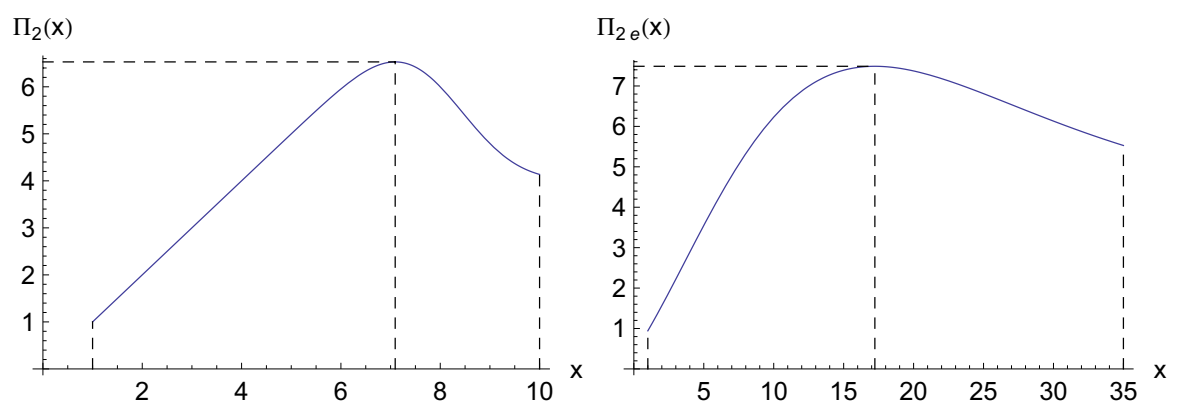

FIGURE 4. Representation of the profit function and optimal solution when the belief distribution of the two manufacturers follows a binormal distribution whose parameters $\{\mu, \sigma\}$ are $\{5,0.5\}$ and $\{8,1\}$ with a correlation coefficient $\rho=0.9$ (left graph); when the belief distribution follows extreme value distributions $\{1,8\}$ and $\{2,10\}$ (right graph); for both $\alpha=4$.

For the binormal distributions, the optimal value is $x^{*}=7.4875$ and in the extreme value ones $x^{*}=17.231$. The plots of the profit functions and corresponding maxima are presented in Figure 4.

\section{Conclusion}

In many supply chains, it is worthwhile to investigate the information available to bargaining firms for improving system-wide performance. The focus of our paper is on the analysis of the static pre-contract period where the supplier makes his first offer. In this paper, we provide our review, discussion and application of the supplier's and manufacturer's behaviours when information about competition or reservation value and demand function is asymmetric information available only to the manufacturer. Under asymmetric information, not only is rent transferred from supplier to manufacturer, but the manufacturer will actively hide or mis-represent this information to the supplier upon initially setting up their relationship. In a setting of inter-temporal commitment, under the assumption that the supplier is better placed to enhance the quality of his final product, we prove that the efficiency of the supply chain is affected because, in the price definition phase, the downstream partner will strive to capture a disproportionate share of the overall rent. With these results, we address an insufficiency in supply chain management literature. All previous models consider that at least part of the information which we consider here to be private is available. In particular, all newsvendor models and those derived from them consider that the supplier knows of the final demand distribution. All the supply chain contracts [2,31] or the explicit production-distribution models [12] consider that, even though demand is stochastic, the supplier knows of its distribution function. 
The present results build upon a large family of distribution functions (uniform, Weibull, extreme value, normal distribution, gamma, exponential, ...) which enjoy the characteristic of being log-concave. The interest of such distributions lies in their ability to represent beliefs about price distributions which range between positive upper and lower finite bounds, a feature which is common to all beliefs about prices.

The analysis presented here also is a first step in understanding and representing the precision of information in game theoretic supply chain models. This feature has never been approached before, except in fuzzy logic models but these do not contemplate the influence of the downstream partner on the precision or accuracy of the information. Further applications of Bayesian beliefs in supply chain settings with asymmetric or inaccurate information can be envisioned. It is left to future research to consider the dynamic multi-period setting in a broader sense with strategic customers able to react to price offers.

Acknowledgements. I would like to acknowledge the precious help in straightening out the kinks in this paper from my colleague Roxane Cattan-Jallet, from Yoav Kerner at Ben Gurion University, from anonymous referees and from discussion at the MS\&OM 2010 conference in Haifa, Israel. All remaining mistakes and inaccuracies are mine.

\section{Appendix A.}

Proof. Proof of Theorem 3.3.

We propose to prove that a unique interior point within the range $[\underline{X}, \bar{X}]$ does indeed maximize it. We first show that the point exists, is a maximum and then prove that it is unique.

\section{A.0.1. Does the optimal interior point exist?}

We now prove that such an optimum exists.

For that, we proceed to prove that

$$
\left\{\begin{array}{l}
\Pi^{\prime}(\underline{X}) \geq 0 \\
\Pi^{\prime}(\bar{X}) \leq 0
\end{array}\right.
$$

By construction of $F($.$) , even though f(x)>0$ and $F(x)<1$, at the limit,

$$
\left\{\begin{array}{l}
\lim _{x \rightarrow \underline{X}} \Pi^{\prime}(x)=f(\underline{\underline{X}})(\alpha-\underline{X})+1 \\
\lim _{x \rightarrow \bar{X}} \Pi^{\prime}(x)=f(\overline{\bar{X}})(\alpha-\overline{\bar{X}}) .
\end{array}\right.
$$

For both conditions in (A.1) to be true, we obtain the following conditions

$$
\left\{\begin{array}{l}
\underline{X}<\alpha+\frac{1}{\bar{X}(\underline{X})} \\
>\alpha
\end{array}\right.
$$




\section{A.0.2. Is the optimum a maximum?}

A property of the increasing failure rate which is of interest in what follows is that

$$
r^{\prime}(x) \geq 0
$$

This means that

$$
f^{\prime}(x)(1-F(x))+f(x)^{2} \geq 0 .
$$

The first order condition (F.O.C.) requires that

$$
\Pi^{\prime}(x)=f(x)(\alpha-x)+\bar{F}(x)=0 .
$$

We describe in the following corollaries the properties of this first differential

For all cases such that $f(x)>0$, we can write the F.O.C. as

$$
\alpha-x=-\frac{\bar{F}(x)}{f(x)} .
$$

The second order condition (S.O.C.) for a maximum requires that

$$
\Pi^{\prime \prime}(x)=(\alpha-x) f^{\prime}(x)-2 f(x)<0
$$

In the case when $f(x)>0$, when we replace $(\alpha-x)$ from (A.7) in (A.8), we obtain

$$
f(x) \Pi^{\prime \prime}(x)=-r^{\prime}(x)-f(x)^{2} .
$$

Since $f($.$) is positive and r($.$) is increasing, when the F.O.C. is satisfied, the S.O.C.$ is also satisfied.

By definition of the first differential of the failure rate $r^{\prime}(x) \geq 0$. So, because $f(x)>0, R^{\prime \prime}(x)<0$.

So, if

$$
\exists x_{0} \mid \Pi^{\prime}\left(x_{0}\right)=0 \Rightarrow \Pi^{\prime \prime}\left(x_{0}\right)<0 .
$$

If a value exists which is an extremum for the objective function, it is a maximum.

Let us now see whether this maximum is unique.

\section{A.0.3. Is this maximum unique?}

Reasoning by the absurd, if

$$
\exists\left(x_{0}, x_{1}\right) \in[\underline{X}, \bar{X}]^{2}, \mid x_{0}<x_{1}, \Pi^{\prime}\left(x_{0}\right)=\Pi^{\prime}\left(x_{1}\right)=0,
$$

then by (A.10),

$$
\Pi^{\prime \prime}\left(x_{0}\right)<0 \wedge \Pi^{\prime \prime}\left(x_{1}\right)<0 .
$$


Since $\Pi($.$) is continuous by construction, it decreases for values in the vicinity$ and above $x_{0}$, whereas it increases for values in the vicinity but below $x_{1}$. Hence, between $x_{0}$ and $x_{1}, R^{\prime}($.$) changes sign, so that$

$$
\left.\exists x_{2} \in\right] x_{0}, x_{1}\left[, \mid \Pi^{\prime}\left(x_{2}\right)=0, \Pi^{\prime \prime}\left(x_{2}\right) \geq 0,\right.
$$

This contradicts (A.10). Hence there cannot exist another point $x_{1}$, distinct from $x_{0}$, for which $\Pi^{\prime}\left(x_{1}\right)=0$.

We conclude that the point which represents the maximum of the objective function in the interval $[\underline{X}, \bar{X}]$, if it exists, is unique.

\section{A.1. Extending to Results to AlL LOG-COnCAVE Distributions}

In this section, we present some useful properties of the IFR distributions. We show some properties of the multivariate distribution functions. The multiplication by a scalar can allow a principal involved in a multi period game to update his belief.

We list here some of the properties which are of interest in the following demonstrations.

From Theorem 4.1 on page 25 of [4], the following statements are equivalent. We assume $F\left(0^{-}\right)=0$.

- $\mathrm{F}$ is an IFR distribution.

- $\log \bar{F}(t)$ is concave for $t$ in $\{t \mid F(t)<1, t \geq 0\}$.

- $\bar{F}(t)$ is $\mathrm{PF}_{2}^{8}\left(\bar{F}(x+y)\right.$ is $\mathrm{TP}_{2}$ in $x$ and $y$ for $\left.x+y \geq 0\right)$.

Of interest to us here, Theorem 1 on page 446 and Theorem 3 on page 448 from [3] enounce the following. Let $f$ be a probability density function whose support is the interval $(a, b)$, and let $\bar{F}$ be the corresponding reliability function:

- If the density function $f$ is continuously differentiable and log-concave on $(a, b)$, then both $F,(\bar{F})$ is also log-concave on $(a, b)$.

- If $F,(\bar{F})$ is log-concave on $(a, b)$, then the right hand integral $H$ of the reliability function, defined by $H(x)=\int_{x}^{b} F(t) \mathrm{d} t,\left(H(x)=\int_{x}^{b} \bar{F}(t) \mathrm{d} t\right)$, is also log-concave on $(a, b)$.

Corollary 2 from the same source is also of interest here:

If the density function $f$ is log-concave on $(a, b)$, then the failure rate $r(x)$ is monotone increasing on $(a, b)$.

Reference [3] in Remark 1, which states that the converse of the Corollary 2, is not true because there exist probability distributions with monotone increasing failure rates but no log-concave density functions might be considered to be limiting. However, this remark does not concern us here because it involves distributions with negative left tails, whereas we limit our interest to distributions with finite positive supports: $(a, b),\{a, b\} \in \mathbb{R}^{2+}$.

\footnotetext{
${ }^{8}$ Pólya frequency density function of order 2 .
} 
In fact, as recorded by [1], for a positive-valued random variable, when its distribution is log-concave, then its failure rate is weakly increasing. Further, these distributions have finite moments of all orders [4]. This result is derived through a comparison to the exponential distribution, which has a constant failure rate.

Another interesting property is that if $X$ is IFR, then so is $\delta+\nu X$ for constants $\delta, \nu>0[28]$.

We conclude that all log-concave distributions for positive-valued random variables have increasing failure rates. Another conclusion which can be drawn is that the supplier may use any log-concave distribution function for his belief and still obtain a unique profit-maximising offering price.

\section{REFERENCES}

[1] Mark Yuying An, Logconcavity versus logconvexity: a complete characterization. J. Econ. Theory 80 (1998) 360-369.

[2] R. Anupindi and Y. Bassok, Supply contracts with quantity commitments and stochastic demand, in Quantitative models for supply chain management, edited by S. Tayur, R. Ganeshan, and M. Magazine. Kluwer Academic, Dordrecht, Holland (1998) 197-232.

[3] M. Bagnoli and T. Bergstrom, Log-concave probability and its applications, Econ. Theory 26 (2005) 445-469.

[4] R.E. Barlow and F. Proschan, Mathematical theory of reliability. John Wiley \& Sons (1965) Chap. 1, 9-18,

[5] D. Baron and D. Besanko, Regulation and information in a continuing relationship. Inf. Econ. Policy 1 (1984) 267-302.

[6] X. Brusset, Multi-period contracts in transport under asymmetric information and prior investments, in Logistik management, systeme, methoden, integration, edited by S. Voß, J. Pahl and S. Schwarze. Business and Economics, Springer-Verlag Berlin, Heidelberg (2009) $37-54$.

[7] X. Brusset, Modeling contractual relationships in transport, Ph.D. Thesis, Louvain School of Management, Louvain la Neuve, Belgium (2010).

[8] G. Cachon, Supply chain coordination with contracts, in Handbooks in operations research and management science: Supply chain management, edited by Ton de Kok and S. Graves, Vol. 11, Elsevier (2004) 229-340.

[9] G. Cachon and Martin Lariviere, Contracting to assure supply: how to share demand forecasts in a supply chain. Manage. Sci. 47 (2001) 629-646.

[10] G. Cachon and S. Netessine, Game theory in supply chain analysis, in Handbook of quantitative supply chain analysis - modeling in the eBusiness era, 1 ed., International Series in Operations Research \& Management Science. Springer Science, New York, NY (2004) Chap. 2, 13-66.

[11] F. Chen, Information sharing and supply chain coordination, in Handbooks in operations research and management science: Supply chain management, edited by T. de Kok and S. Graves, Vol. 11, Elsevier (2004) 341-421.

[12] Z.-L. Chen, Handbook of quantitative supply chain analysis, in Integrated production and distribution operations, Kluwer Academic Publishers (2004), Chap. 17, 711-745.

[13] Wai Hung J. Chu and Ching Chyi Lee, Strategic information sharing in a supply chain. Eur. J. Oper. Res. 174 (2006) 1567-1579.

[14] Ch. Corbett, Stochastic inventory systems in a supply chain with asymmetric information: Cycle stocks, safety stocks, and consignment stock. Oper. Res. 49 (2001) 487-500.

[15] Ch. Corbett and X. de Groote, A supplier's optimal quantity discount policy under asymmetric information. Manage. Sci. 46 (2000) 444-450. 
[16] Ch. Corbett and Ch. Tang, Designing supply contracts: contract type and information asymmetry, in Quantitative models for Supply Chain Management, edited by S. Tayur, R. Ganeshan and M. Magazine. Kluwer Academic, Dordrecht, Holland (1999) 234-267.

[17] D. Fudenberg and J. Tirole, Game theory, Chap. Bayesian games and mechanism design. MIT Press (1991) 243-318.

[18] R. Gibbons, Game theory for applied economists, Princeton University Press, Princeton, NJ, USA (1992).

[19] R. Gibbons, An introduction to applicable game theory. J. Econ. Perspect. 11 (1997) 127149.

[20] Albert Y. Ha, Supplier-buyer contracting: Asymmetric cost information and cutoff level policy for buyer participation. Nav. Res. Logist. 48 (2001) 41-64.

[21] J. Harsanyi, Games with incomplete information played by "Bayesian" players. Manage. Sci. 14 (1967) 159-182, 320-334, 486-502.

[22] J.C. Harsanyi, Games with incomplete information played by "Bayesian" players, i-iii. Management Sci. 50 (2004) 1804-1817.

[23] M. Lariviere, Supply chain contracting and coordination with stochastic demand, in Quantitative models for supply chain management, edited by S. Tayur, R. Ganeshan and M. Magazine. Kluwer Academic, Dordrecht, Holland (1999) 234-267.

[24] M. Lariviere, A note on probability distributions with increasing generalized failure rates. Oper. Res. 54 (2006) 602-604.

[25] M.A. Lariviere and E.L. Porteus, Selling to the newsvendor: an analysis of price-only contracts. Manuf. Serv. Oper. Manag. 3 (2001) 293-305.

[26] M. Leng and M. Parlar, Game theoretic applications in supply chain management: a review. Information Systems Oper. Res. 43 (2005) 187-220.

[27] M. Mesterton-Gibbons, An introduction to game-theoretic modelling. Addison Wesley, Redwood City, California, USA (1992).

[28] A. Paul, A note on closure properties of failure rate distributions. Oper. Res. 53 (2005) 733-734.

[29] A. Paul, On the unimodality of the manufacturer's objective function in the newsvendor problem. Oper. Res. Lett. 34 (2006) 46-48.

[30] A. Rubinstein, Perfect equilibrium in a bargaining model. Econometrica 54 (1982) 97-109.

[31] A. Tsay, S. Nahmias and N. Agrawal, Quantitative models for supply chain management, 1 ed., Int. Ser. Operat. Res. Manage. Sci., in Modeling supply chain contracts: A review. Kluwer Academic (1999), Chap. 10, 299-336.

[32] W. Vickrey, Counterspeculation, auctions, and competitive sealed tenders. J. Finance 16 (1961) 8-37.

[33] S. Ziya, H. Ayhan and R.D. Foley, Optimal pricing for a service facility, Working paper, in proc. School of Industrial and Systems Engineering, Georgia Institute of Technology, Atlanta, GA (2004).

[34] S. Ziya, H. Ayhan and R.D. Foley, Relationships among three assumptions in revenue management. Oper. Res. 52 (2004) 804-809. 